\title{
Calidad de los Servicios prestados por el Servicio Autónomo Municipal de Administración Tributaria
}

\author{
Clemenza, Caterina * \\ Gotera, Ana ** \\ Araujo, Rubén ***
}

\section{Resumen}

El presente estudio tiene como objetivo analizar la calidad de servicio prestado por el Servicio Autónomo Municipal de Administración Tributaria (SAMAT), a través de confrontar percepción y las expectativas de los contribuyentes marabinos referente al servicio ofrecido por el SAMAT. La investigación fue de tipo descriptivo con diseño no experimental, transeccional y de campo. A tal fin se desarrollo un instrumento tipo cuestionario aplicado a una muestra de 270 contribuyentes, en tres periodos característicos para el sector de estudio. El instrumento fue validado por cinco expertos, luego se procedió a calcular la confiabilidad a través del método Alfa de Cronbach cuyo coeficiente se ubico en 0,97 . Se concluye que la calidad de servicio percibida cubre medianamente las expectativas de los clientes en cuanto a los componentes analizados (elementos tangibles e intangibles: fiabilidad, capacidad de respuesta, seguridad, empatía); de manera tal que los contribuyentes no se encuentran satisfechos con el servicio ofrecido por el SAMAT. Se recomienda la creación y puesta en práctica de estrategias que se orienten al desarrollo de un servicio orientado al contribuyente, centrado en atender las expectativas y necesidades del mismo, así como campañas que incentiven al fortalecimiento de una cultura tributaria en el ciudadano.

Palabras clave: Calidad de servicio, expectativas, percepción, contribuyentes tributarios, administración tributaria municipal.

Recibido: 15-06-08. Aceptado: 26-05-09

* Doctora en Ciencias Gerenciales. Profesora titular, adscrita al Instituto de Investigaciones de la Facultad de Ciencias Económicas y Sociales de la Universidad del Zulia. Acreditada al Premio de Promoción al Investigador (PPI- ONCTI). Editora de la Revista de Ciencias Sociales de LUZ. Teléfono.02617596943. E-mail: caterinaclemenza@yahoo.es. Autor para la correspondencia.

** Magíster en Gerencia de Empresas. Mención Gerencia en Mercadeo. E-mail: anagotera21@hotmail.com

*** Doctor en Ciencias Gerenciales. Economista, Comunicador Social. Profesor-Investigador del Núcleo Costa Oriental del Lago de la Universidad del Zulia Acreditado al PPI -ONCTI.

E-mail: raraujove@yahoo.es 


\title{
Quality of services rendered by the Muncipal Autonomous Tax Administration Service
}

\begin{abstract}
The objetive of this study is to analyze the quality of service rendered by the Muncipal Autonomous Tax Administration Service (SAMAT), by comparing perception and expectations of Maracaibo taxpayers regarding the service rendered by SAMAT. Research was of the descriptive type with a non-experimental, trans-sectional, field design. A questionnaire-type instrument was designed and applied to a sample of 270 taxpayers in three periods characteristic for the sector under study. The instrument was validated by five experts, then its reliability was calculated using the Alpha Cronbach method, whose co-efficient was 0.97 . Conclusions were that the perceived service quality only moderately covers clients' expectations in terms of the analyzed components (tangible and intangible elements: reliability, response capacity, security, empathy); therefore, taxpayers are not satisfied with the service offered by SAMAT. Recommendations are that strategies be created and put into practice oriented toward developing a service focused on the taxpayer, centered on responding to his or her expectations and needs, as well as carrying out campaigns to incentivate a taxpaying culture among citizens.
\end{abstract}

Key words: Quality of service, expectations, perception, taxpayers, municipal tax administration.

\section{Introducción}

En Venezuela desde 1992, se han realizado adelantos en materia de reforma a todos los niveles de la administración pública, entendida ésta como el instrumento de ejecución de las políticas del estado para dar respuesta a las crecientes demandas emanadas de la sociedad civil y así lograr el desarrollo económico y social del país.

En este sentido, el estado venezolano ha realizado grandes esfuerzos con el fin de modernizar los órganos de la administración pública y específicamente la administración tributaria que tiene a su cargo la determinación y la recaudación de tributos gravados por los ingresos de los contribuyentes.

En el municipio Maracaibo, del Estado Zulia, a partir de la década del noventa, se unificaron esfuerzos para sol- ventar las dificultades fiscales que confrontaba la administración tributaria municipal representada por la Dirección de Rentas Municipales, en relación al sistema de recaudación de impuestos, enfrentando con estrategias de modernización y actualización los altos índices de evasión y elusión fiscal a fin de fortalecer los ingresos fiscales del Municipio Maracaibo.

Por consiguiente, surge en el año 2001 el proceso de modernización, buscando disminuir la caída de los ingresos por concepto de impuestos, en virtud de que en el año 2000 el Municipio dejó de percibir más de 6 millardo de bolívares, a partir del mes de agosto del año 2001, el Servicio Autónomo Municipal de Administración Tributaria (SAMAT) sustituyó a la Dirección de Rentas Municipales.

El objeto de este organismo es administrar, fiscalizar y aplicar sanciones y resguardar el sistema de los ingresos tri- 
butarios del municipio Maracaibo y los ingresos delegados, con excepción de los relativos a las tasas atribuidas a otros organismos municipales, pero todas estas funciones enmarcadas dentro de un servicio integral de alta calidad, donde todos los procesos sean prestados dentro de una calidad de servicio que facilite al contribuyente el cumplimiento voluntario de sus obligaciones tributarias. Por lo anteriormente descrito, se plantea el objetivo de la presente investigación referido a analizar la calidad de los servicios prestados por el Servicio Autónomo Municipal de Administración Tributar (SAMAT).

El problema se plantea en virtud, de que dicho organismo debe realizar la recaudación de los tributos municipales, los cuales garantizaran la captación de recursos económicos para la Corporación Alcaldía de Maracaibo; tomando en consideración que todas las operaciones de este ente deben ser gestionadas a través de un servicio integral de alta calidad, enmarcando todos los procesos dentro de una calidad de servicio que facilite al contribuyente el cumplimiento voluntario de sus obligaciones tributarias, a través de operaciones confiables, responsables, seguras, con una atención cuidadosa en medio de unas instalaciones modernas y que satisfagan las necesidades de los marabinos.

Tomando en cuenta que a partir del presente año todas las obligaciones tributarias deben ser realizadas por los propios contribuyentes de manera directa y personal ante las oficinas del Servicio Autónomo Municipal de Administración Tributaria; de igual manera se inicia en el 2006 la conexión en línea para el pago de los tributos a través de internet, con el fin de desarrollar el respeto, la confiabilidad y en general la aceptación social del contribuyente.

Por todas estas razones las actividades del Servicio Autónomo Municipal de Administración Tributaria deben desarrollarse a través de la prestación de un servicio fundamentado en la calidad, que satisfaga las necesidades de los contribuyentes y de esta manera resulte mas efectiva la administración de los ingresos tributarios, utilizando a la calidad de servicio prestado por el organismo como elemento clave de una administración justa, moderna, operativa y confiable.

Partiendo de estos argumentos se tiene como propósito analizar la calidad de servicio prestado por el Servicio Autónomo Municipal de Administración Tributaria (SAMAT). Para ello, se desarrollan los siguientes objetivos específicos: identificar los servicios que ofrece el Servicio Autónomo Municipal de Administración Tributaria; determinar las expectativas y la percepción de los contribuyentes con respecto a los elementos tangibles e intangibles que sirven de apoyo al proceso de servicio y establecer la satisfacción de los contribuyentes con respecto a la calidad de servicio prestado por el Servicio Autónomo Municipal de Administración Tributaria.

En tal sentido se desarrolló una investigación de tipo descriptivo, ya que a través de la observación de la variable de estudio, representada por la calidad de servicio prestado por el Servicio Autónomo Municipal de Administración Tributaria, se analiza la calidad de servicio confrontando las expectativas de los usuarios con las percepciones, así como también se determina el grado de conocimiento y de uso con respecto a la variedad de servicios ofreci- 
Calidad de los Servicios prestados por el SAMAT

Clemenza, Caterina; Gotera, Ana y Araujo, Rubén

dos por el ente tributario. Se considera un estudio de campo, ya que en la recolección de los datos se estableció un lugar específico, del cual se tomó la información directamente de fuentes primarias o sujetos de la investigación.

El universo estuvo constituido por todos los contribuyentes tributarios del Municipio Maracaibo, Del mismo modo, la población estuvo conformada por las características observadas y medidas en los contribuyentes tributarios del Municipio Maracaibo, representada por 115.257 contribuyentes, según cifras aportadas por el Servicio Autónomo Municipal de Administración Tributaria (SAMAT, 2007). Siendo la muestra definitiva de 270 contribuyentes, para establecer la muestra de estudio, se utilizó, el muestreo probabilística, aleatorio simple.

A su vez, luego de seleccionar el número de personas a encuestar, se determinó, aplicar el instrumento en tres periodos característicos para el sector de estudio: Un primer periodo comprendido entre el 01 de Julio al 15 de Julio, periodo de declaración, con un $10 \%$ de descuento por pronto pago, periodo en el cual se aplicara el instrumento a 90 contribuyentes de la muestra.

Un segundo periodo comprendido entre el 15 de Julio al 31 de Julio periodo de declaración, sin descuento, de igual manera se recogerá la información a 90 contribuyentes. $Y$ un último periodo fuera del lapso de declaración de SAMAT, donde se aplicara el instrumento a 90 contribuyentes, debido a que las jornadas de declaración solo se presentan cuatro veces al año, por lo tanto la mayor parte del tiempo está fuera de jornadas. Todo esto con el fin de que el estudio presente unos resultados respaldados por características particulares que influirán tanto en las expectativas como en las percepciones del servicio.

\section{Consideraciones teóricas sobre marketing de servicio}

Desde mediado de los años noventa, el sector servicio ha crecido con rapidez, gracias al proceso de globalización que le ha permitido modernizarse y desarrollarse, al incorporarse diversos segmentos de servicio, entre los cuales se pueden mencionar comidas rápidas, servicios de telecomunicaciones, financieros, de salud privado, entre otros; todo ello ha inducido a una mayor competencia exigiéndole a las empresas de servicios "adapten una posición más agresiva para conservar y conquistar nuevos espacios" (Cobra, 2001:17).

Para Kotler, Armstrong, Cámara y Cruz (2004) los negocios de servicios son más difíciles de administrar, empleando solo el enfoque tradicional de comercialización, ya en las organizaciones de servicio, el cliente interactúa con el prestador del mismo, además con todo el proceso que sustenta su producción. Por tanto, la comercialización del servicio requiere enfocarse hacia la mercadotecnia interna y la mercadotecnia interactiva. Entendiéndose por la primera como el esfuerzo de la organización para capacitar y motivar en forma eficaz a los empleados quienes tienen contacto con los clientes y del personal de apoyo con el fin de proporcionarle satisfacción y pueda complacer los requerimientos del cliente.

Al respecto Zeithaml y Bitner (2002) señalan que los proveedores de- 
ben contar con herramientas y motivaciones las cuales permiten prestar efectivamente el mismo, mientras que la mercadotecnia interactiva se enfoca en la calidad de interacción entre el comprador y el vendedor; es decir, dependerá del prestador del servicio como de la calidad del servicio prestado.

Para Zeithaml y Bitner (2002), el marketing interactivo ocurre en el momento de la verdad, es decir, cuando el cliente interactúa con la organización y el servicio se produce y consume. Se debe destacar que cada vez que el cliente interactúa con el servicio, las promesas se cumplen o se rompen y la confiabilidad del servicio se pone a prueba.

En este sentido, administrar la calidad de los servicios, significa que las organizaciones describan que los servicios de excelente calidad les da una fuerte ventaja competitiva; para ello deben esforzarse por superar las expectativas de sus clientes.

Lo cual conduce a elaborar estrategias distintivas para satisfacer las necesidades de los clientes, usar métodos para medir la opinión de los clientes con respecto al servicio, comunicar las preocupaciones sobre la calidad de los mismos a todos los integrantes de la organización y mantener buenas relaciones con los empleados, recompensando su buen desempeño y vigilando su satisfacción laboral.

Todos estos aspectos diferenciados permiten agregarle valor a fin de que el servicio percibido por los clientes de una organización supere el esperado y éstos vuelvan a recurrir al proveedor, lo cual conduce a que la organización conserve sus clientes.

Al hablar de matriz de servicios, se debe hacer referencia a la mezcla del mis- mo, para ello, Zeithaml y Bitner (2002), reconocen que además de los cuatro "Ps" tradicionales, la mezcla de marketing de servicio incorpora a las personas, las evidencias físicas y el proceso. Donde las personas prestadoras del servicio transmiten ciertos mensajes a los clientes e influyen sobre sus percepciones.

Pero no solo el prestador directo forma parte de este grupo de personas, sino también los clientes quienes pueden influir en la entrega del servicio, al respecto los clientes no solo tienen influencia en el resultado de su propio servicio, sino también en el de los otros.

En el teatro, un juego de pelota o un salón de clases, los clientes pueden influir sobre la calidad del servicio que reciban de los demás bien sea para mejorar o desmerecer la apariencia de los otros clientes" (Zeithaml y Bitner, 2002: 25).

La evidencia física se refiere al ambiente tangible donde se interactúa, desempeña o comunica el servicio. Los elementos de las evidencias físicas constituyen excelentes oportunidades para que las empresas transmitan mensajes consistentes y sólidos en relación con el propósito de la organización, los segmentos del mercado que pretende y la naturaleza del servicio (Zeithmal y Bitner, 2002: 25).

Entre los elementos de la evidencia física se tiene el interior y exterior de las instalaciones, diseño de exterior estacionamientos, señalizaciones, calidad del aire, temperatura, papelería estado de la facturación, apariencia física de los empleados, entre otros.

Todos los elementos antes mencionados (personas, evidencia físicas y procesos) se encuentran bajo el control de la empresa y todos ellos individual- 
Calidad de los Servicios prestados por el SAMAT

Clemenza, Caterina; Gotera, Ana y Araujo, Rubén

mente o en conjunto pueden influir en la decisión de compra del cliente, así como sobre su satisfacción y sobre la decisión de adquirir nuevamente el servicio.

\section{Calidad del servicio}

Antes de comenzar a discutir sobre la calidad del servicio, se hace necesario definir calidad. A lo largo de la historia, el término calidad ha sufrido numerosos cambios, convirtiéndose en uno de los requisitos esenciales y un factor clave del cual depende la mayor parte de la organización, no solo para mantener su posición en el mercado sino incluso para asegurar su supervivencia.

La calidad se define usualmente como un término subjetivo donde cada persona tiene su propia definición. Técnicamente la calidad tiene dos significados: las características de un producto o servicio con habilidades para satisfacer necesidades explícitas o implícitas; o un producto o servicio libre de defectos.

Para Duque (2005), cuando se conceptualiza la calidad en su sentido más general, se hace referencia al nivel elegido para satisfacer cada uno de sus usuarios y se va acercando a la excelencia cuando responde a las necesidades y expectativas de dichos usuarios.

Por tanto, el concepto de calidad en un ambiente de la administración de la calidad total, se basa en servir a los clientes al máximo grado posible, donde los servicios o productos satisfagan los requerimientos y necesidades de los mismos. Es el cliente quien juzga si la calidad de los bienes y servicios es aceptable para satisfacer sus necesidades, entonces, es éste quien debe ser el centro de cualquier organización de servicio que busque la excelencia y lealtad de sus clientes.

La calidad puede ser vista como excepción o especial, dentro de las organizaciones; considerándola, en primer lugar como diferente, de clase superior y otorgándole un sentido de exclusividad. En segundo lugar como equivalente a excelencia, o el logro de un estándar muy alto, pero en circunstancias muy limitadas, dependiendo por lo general, por la reputación de la institución. Y en tercer lugar implica alcanzar estándares mínimos.

También puede asumirse como perfección asegurándose la carencia de errores. Aún más, este enfoque exige que dicha perfección sea entregada de manera consistente basada en concepción de cero defectos, y la de hacer las cosas bien. En cuanto a cero defectos intrínsicamente está ligado a la noción de cultura de calidad. En una cultura de calidad todos en la organización son igualmente responsables del producto final.

Por otra parte, hacer las cosas bien implica que no hay errores en ninguna etapa del proceso, lo cual incorporado a productos (bienes y servicios) y procesos se acerca al concepto de calidad total.

La calidad como aptitud para el logro de un propósito implica la relación con la forma como cierto producto o servicio se ajusta a un propósito.

La calidad como valor agregado se ha estado usando desde mediados de los años ochenta en varios países donde el producto generado es enriquecido en todas sus fases. En este enfoque subyace el concepto de "accountability" (obligación de rendir cuentas), donde las organizaciones son responsables de la calidad 
ante los organismos que las financian y los clientes.

Según Duque (2005) la calidad se puede definir de diferentes maneras, siendo las más comunes, las siguientes:

1. La calidad entendida como la consistencia del producto, se encuentra más cercana al ámbito de la industria donde la búsqueda de métodos para reducir al mínimo los defectos en el producto o el servicio ha sido una de las banderas del pensamiento de la calidad total.

2. La calidad concebida como el cumplimiento de una misión, donde se está ante el concepto de eficacia y eficiencia en el cumplimiento de objetivos previamente trazados.

3. La calidad definida como la satisfacción de las necesidades del cliente, está relacionada directamente con mercado, que premia el valor recibido a cambio de una inversión.

Así, la búsqueda de la calidad en las organizaciones, permite desarrollar sus capacidades, a fin de que los productos y servicios tengan condiciones diferenciadas para su uso, actuando de acuerdo a técnicas y procedimientos para la planeación, control y mejoramiento de todas las actividades organizacionales, con el propósito de ofrecerle al cliente un producto o servicio orientado a satisfacer sus necesidades y superar sus expectativas.

Cuando se trata de bienes manufacturados, la calidad de los productos puede ser evaluada a través de las especificaciones concretas previamente establecidas; por el contrario evaluar el servicio resulta un tanto más difícil debido a su naturaleza intangible, requiriéndose establecer indicadores objetivos de la cali- dad del servicio, lo cual depende de la percepción, expectativas y necesidades de los clientes frente al servicio recibido.

La calidad en el servicio implica un nivel de excelencia tal que cubra las necesidades de sus usuarios, así como alcanzar una satisfacción del mismo. Es decir, el servicio alcanza el nivel de excelencia cuando responde a las demandas de los receptores del mismo.

Para Zeithaml y Bitner (2002), la calidad de servicio es la amplitud de la discrepancia o diferencia que existe entre las expectativas o deseos de los usuarios y sus percepciones.

La calidad en el servicio implica lograr un nivel de excelencia tal que cubra las necesidades de sus usuarios, así como alcanzar una satisfacción del mismo. Se convierte en un instrumento que brinda una ventaja significativa para la institución.

Al respecto, Berry (2004), considera que el éxito en las organizaciones es el resultado de los activos fijos, de los recursos de infraestructura, y de los activos intangibles, representado por las capacidades y conocimientos de las personas que laboran allí, así como también de las actividades del buen servicio al usuario.

Si bien es cierto que la calidad asegura la continuidad, mejora los métodos de trabajo y aumenta la satisfacción laboral, se requiere del compromiso de todos los integrantes de la organización para poder lograr excelencia en el servicio que puedan satisfacer las expectativas de los usuarios.

Para Zeithaml y Bitner (2002), una forma de transmitir la impresión de un buen servicio, es que parezca una operación de calidad, prestando atención a 
Calidad de los Servicios prestados por el SAMAT

Clemenza, Caterina; Gotera, Ana y Araujo, Rubén

los detalles para impresionar al usuario actual, al usuario potencial y a los empleados.

Por su parte Berry (2004) asevera que la calidad de servicio es la filosofía basada en los principios del liderazgo con espíritu colectivo de servicio proporcionando maneras eficaces para conservar a los clientes; es decir, se ha constituido en la base fundamental para conservar al cliente y lograr que éste sea constante con aquellas organizaciones prestadoras de un servicio con criterio de calidad. Bajo este enfoque se busca la satisfacción total y completa del usuario; para ello la organización deberá realizar esfuerzos importantes a fin de conocer sus necesidades y expectativas.

En este sentido, en las organizaciones actuales se hace imprescindible ofrecer un servicio de calidad a fin de proyectar una imagen positiva permanente en el tiempo y se convierta en una variable que influya y determine las conductas, actitudes y opiniones del público. Al respecto, resulta fundamental el conocimiento de las características de los servicios que se ofrecen, la satisfacción de los clientes y la imagen de la organización. Por ello es tan importante el constante análisis de todos estos aspectos, pues constituye un grave problema desconocerlos, considerando que no atenderlos y mejorarlos permanentemente puede generar consecuencias negativas para las organizaciones (Berry, 2004).

\section{Métodos para evaluar la calidad del servicio}

Se han desarrollado varios métodos con el fin de determinar la calidad del servicio percibida por el cliente. Estos métodos varían de acuerdo a las corrientes teóricas o escuelas que los promueven, entre ellas la Escuela europea o nórdica, la cual se encuentra liderada por Growoos $(1988,1994)$, quien formuló el modelo conocido como "Modelo de la Imagen", donde relaciona la calidad del servicio con la imagen corporativa.

Plantea tres dimensiones básicas: la técnica, la funcional y la imagen; por lo tanto argumenta que la calidad percibida por los clientes es la integración de la calidad técnica (qué se da) y la calidad funcional (cómo se da), las cuales se relacionan con la imagen corporativa, donde la imagen es el elemento básico para medir la calidad percibida.

Aquí, solo la dimensión imagen presenta rasgos proyectados hacia el exterior de la organización; mientras, las otras dos (técnica y funcional), son elementos internos de la organización; donde la calidad técnica se enfoca en un servicio técnicamente correcto, conduciendo a un resultado aceptable; y la calidad funcional se encarga de la manera como el cliente es tratado en el desarrollo del proceso de producción del servicio, es decir, como el cliente recibe el servicio; y la imagen corporativa es la forma como el cliente percibe la calidad técnica y la funcional.

De acuerdo a este criterio, la calidad total percibida, está dada por las diferencias existentes entre la calidad esperada y la experimentada, mas que por el nivel objetivo de las dimensiones técnicas y funcionales (Duque, 2005; Bellido, 2004).

Otra es la escuela americana, quien postula el modelo SERVQUAL y SERVPERF. En la literatura referida a la medición de la calidad del servicio, el mo- 
delo con mayor aceptación y difusión es el SERVQUAL, propuesto por Parasuraman, Zeithaml y Berry en 1988, donde se define la calidad del servicio como una función de la discrepancia entre las expectativas de los clientes sobre el servicio a recibir y sus percepciones sobre el servicio efectivamente recibido. Así, un cliente valorará positiva o negativamente la calidad de un servicio cuando sus percepciones sean superiores o inferiores a las expectativas previas.

Este instrumento les permitió aproximarse a la medición mediante la evaluación por separado de las expectativas y percepciones de un cliente, apoyándose en los comentarios hechos por los consumidores en la investigación. Estos comentarios apuntaban hacia diez dimensiones, inicialmente definidas así: Elementos tangibles, Fiabilidad, Capacidad de respuesta, Profesionalidad, Cortesía, Credibilidad, Seguridad, Accesibilidad, Comunicación y Compresión del cliente (Duque, 2005; Hoffman y Bateson, 2002; Zeithaml y Bitner, 2002).

Luego de las críticas recibidas, redefinieron las dimensiones señaladas a través de estudios estadísticos, encontrando correlaciones entre las dimensiones iniciales, que a su vez permitieron reducirlas a cinco.

Definitivamente, el modelo quedó conformado por la existencia de cinco (5) dimensiones de calidad del servicio: elementos tangibles, confiabilidad, responsabilidad, seguridad y empatía, tanto para la percepción como para las expectativas, las cuales son medidas a través de 44 aseveraciones (22 para las expectativas y 22 para las percepciones) iguales tanto para las expectativas como para las percepciones en escala Likert de siete (7) puntos., luego se comparan los resultados de las dos secciones para obtener las calificaciones de las dos brechas, de cada una de las cinco dimensiones.

Cuanto más grande sea la brecha, más alejadas estarán las percepciones de las expectativas y más baja será la evaluación de la calidad del servicio. Al contrario, cuanto menor sea la brecha mayor será la evaluación de la calidad del servicio (Duque, 2005; Hoffman y Bateson, 2002; Zeithaml y Bitner, 2002).

También plantean el hallazgo de una serie de vacíos, desajustes o gaps en el proceso. Estos influyen en la percepción del cliente y son el objeto de análisis cuando se desea mejorar la calidad percibida. Así, las percepciones generales de la calidad de servicio están influidas por estos vacíos (gaps) que tienen lugar en las organizaciones que prestan servicios. Definen vacío o gap como una serie de discrepancias o deficiencias existentes respecto a las percepciones de la calidad de servicio de los ejecutivos y las tareas asociadas con el servicio que se presta a los consumidores.

En cuanto al modelo SERVPERF, este fue ideado por Cronin y Taylor, en 1992; quienes establecieron una escala más concisa que SERVQUAL. Esta nueva escala está basada exclusivamente en la valoración de las percepciones de los clientes, con una evaluación similar a la escala SERVQUAL, pero sin tomar en cuenta las expectativas de los clientes, argumentándose una tendencia generalizada a darle altas valoraciones a las expectativas, siendo las percepciones las cuales más contribuyen a medir la calidad del servicio. Este modelo, aunque es ca- 
Calidad de los Servicios prestados por el SAMAT

Clemenza, Caterina; Gotera, Ana y Araujo, Rubén

paz de medir la calidad del servicio, no puede establecer en cuales elementos es prioridad mejorar la calidad del servicio (Duque, 2005; Bellido, 2004).

Los instrumentos empleados por los modelos SERVQUAL y SERVPERF, se consideran las escalas más importantes para medir la calidad del servicio. Ambos coinciden en que es el cliente quien juzga si un servicio es de calidad.

\section{Dimensiones de la calidad del servicio}

Los clientes perciben la calidad como un concepto que incorpora más de una dimensión; es decir, las valoraciones de los clientes acerca de la calidad se basan en la percepción de múltiples factores. Por ejemplo, se dice que las siguientes ocho dimensiones de la calidad se aplican a todos los productos y servicios: desempeño, características, confiabilidad, adaptabilidad, durabilidad, utilidad, estética y percepción de la calidad (más o menos equivalente a prestigio). Sin embargo, algunos otros expertos han argumentado que las dimensiones de la calidad sólo adquieren significado cuando se aplican en categorías de productos (como productos duraderos, productos empacados, servicios).

Otros investigadores han descubierto que los clientes toman en cuenta cinco dimensiones para valorar la calidad de los servicios, entre ellos: las dimensiones intangibles representados por Fiabilidad o confiabilidad, capacidad de respuesta o responsabilidad, seguridad, empatía y los elementos tangibles tangible. Estas dimensiones representan la forma como los clientes organizan men- talmente la información sobre la calidad en el servicio.

De acuerdo con los hallazgos de una investigación exploratoria y cuantitativa, Zeithaml y Bitner (2002) manifiestan que Parasuraman, Zeithaml y Berry, (1985), establecieron que las cinco dimensiones anteriores son relevantes para los servicios financieros, los seguros, la reparación y el mantenimiento de aparatos domésticos, el corretaje de valores, el servicio telefónico de larga distancia y el servicio de reparación de automóviles, el servicio de hospedaje, entre otros.

\section{Fiabilidad o Confiabilidad.}

Es la capacidad para desempeñar el servicio prometido con formalidad y exactitud. Significa brindar el servicio en forma correcta desde el primer momento. Aquí se incluyen todos los elementos para permitirle al cliente detectar la capacidad y conocimientos profesionales de la organización. En su sentido más amplio, la confiabilidad significa el cumplimiento, por parte de la empresa de sus promesas, lo convenido acerca de la entrega, la prestación del servicio, la solución de problemas y los precios. Los clientes desean hacer negocios con las organizaciones cuando cumplen sus promesas, particularmente las relacionadas con los atributos del servicio básico (Zeithaml y Bitner, 2002).

Capacidad de Respuesta o Responsabilidad.

La responsabilidad se define como la disponibilidad para ayudar a los clientes y para proveer el servicio con prontitud. La responsabilidad es la voluntad de colaborar con los clientes y de prestar el servicio con prontitud. Esta dimensión destaca la atención y la prontitud con para hacerle frente a las solicitudes, las 
preguntas, las reclamaciones y los problemas de los clientes. La responsabilidad se comunica a los clientes a través de un lapso de tiempo donde debe esperar para recibir la asistencia, la respuesta a sus preguntas o la atención de sus problemas, La responsabilidad también comprende la noción de flexibilidad y la capacidad para personalizar el servicio a las necesidades del cliente.

\section{Seguridad}

En esta dimensión, el cliente coloca en manos de la organización sus problemas y siente la certeza de que serán resueltos de la mejor manera posible. La seguridad implica credibilidad, integridad y honestidad. Es probable que esta dimensión adquiera particular importancia para aquellos servicios donde los clientes perciben involucrarse en un gran riesgo o en los cuales se sienten inseguros acerca de su capacidad para evaluar los resultados, por ejemplo los servicios financieros, de seguros, de corretaje, médicos y legales (Zeithmal y Bitner, 2002).

Existen organizaciones, que debido a la naturaleza de sus operaciones, deben procurar construir confianza y lealtad entre las personas de contacto más importantes y los clientes individuales. Esta idea se refleja en el concepto del banquero personal, el cual significa que coordinará todos sus servicios financieros. Uno de los riesgos inherentes que corre la compañía cuando construye este tipo de relaciones personales consiste en que el cliente seguirá al empleado del servicio si éste abandona la compañía (Zeithmal y Bitner, 2002).

Durante las primeras etapas de una relación es posible que el cliente utilice las evidencias tangibles para valorar la dimensión de la seguridad. En este caso las evidencias físicas como los títulos profesionales, los reconocimientos, los premios y los certificados especiales pueden brindar al nuevo cliente la seguridad sobre un determinado proveedor de servicios profesionales.

\section{Empatía}

Es tratar a los clientes como persona. La empatía se define como la atención cuidadosa e individualizada donde la organización le brinda y transmite a sus clientes un servicio personalizado o adecuado y le genera la idea de ser únicos y especiales. Los clientes quieren sentirse importantes comprendidos para las empresas prestadoras el servicio. La organización cuando se conduce bajo esta dimensión requiere desarrollar un fuerte compromiso con el cliente, conociendo al detalle sus características y sus especiales requerimientos (Zeithaml y Bitner, 2002).

\section{Tangibles}

Es la parte visible de la oferta del servicio. Se define como tangible a la apariencia de las instalaciones físicas, el equipo, el personal y los materiales de comunicación. Todos ellos transmiten representaciones físicas o imágenes del servicio que los clientes, y en particular los nuevos, utilizarán para evaluar la calidad.

Es frecuente en las empresas de servicios utilizar los tangibles con el fin de realzar su imagen, proporcionar continuidad e indicar la calidad a los clientes, la mayoría de las empresas combinan los tangibles con otras dimensiones para crear una estrategia de calidad. Las organizaciones que no le dan importancia a los elementos tangibles dentro de sus estrategias de negocios podría menoscabar e incluso destruir las otras estrategias di- 
Calidad de los Servicios prestados por el SAMAT

Clemenza, Caterina; Gotera, Ana y Araujo, Rubén

rigidas a elevar la calidad del servicio (Zeithaml y Bitner, 2002).

Así para efectos de este artículo, se consideran las cinco para que los contribuyentes (usuarios) del SAMAT, valoren la calidad de servicio ofrecida en esta institución municipal.

\section{Resultados del estudio}

A continuación se presentan los resultados obtenidos de la investigación y que se detallan de acuerdo a las dimensiones estudiadas.

a) Servicios ofrecidos por el Servicio Autónomo de Administración Tributaria (SAMAT)

Entre los servicios prestados por el SAMAT, se encuentran, registro de información municipal, pago de impuestos por actividades económicas, comerciales, industriales de servicio y de índole similar, pago de impuestos por propaganda y publicidad comercial, pago de impuestos por espectáculos públicos, pago de impuestos por cementerio, pago de impuestos de vehículos inscripción para la actualización de expendio bebidas alcohólicas, pago de impuestos por juegos y apuestas licitas, pago de impuestos por tasa de servicios de copias y certificaciones de documentos y pago de impuestos por inmuebles urbanos.

En el Gráfico 1, se observa el conocimiento que tienen los usuarios, en cuanto al lugar donde deben pagar sus tributos municipales. En este sentido, el 36,70\% de los contribuyentes indicaron acceder la página web para cancelar los impuestos; el $26,30 \%$, en los centros de atención ubicados en los centros comerciales Sambil y Lago Mall; el 13,35\% señaló que solo conocen las empresas recaudadoras y por tanto allí hacen efectivo su pago y un $8,13 \%$ solo afirmó conocer las taquillas del Banco Occidental de Descuento (BOD). Mientras que un $18,52 \%$ de los contribuyentes desconocían donde debían acudir para pagar sus tributos municipales.

Con estos resultados se evidencia que debe haber una mayor promoción de los lugares donde se deben pagar los impuestos y así los contribuyentes cuenten con conocimiento exacto del lugar donde se deben dirigir para gestionen sus obligaciones según sea el caso.

\section{Gráfico 1}

\section{Conocimiento del lugar para pagar sus tributos}

CONOCIMIENTO DE LOS SERVICIOS PRESTADOS

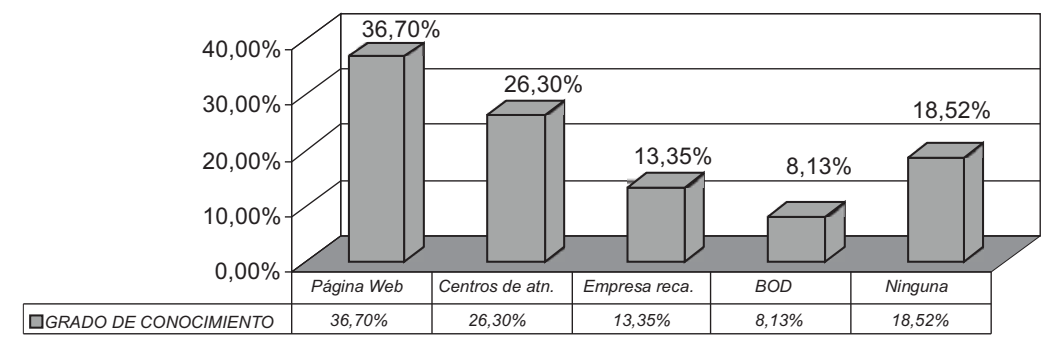

Fuente: Elaboración propia. 
b) Expectativas y Percepción del contribuyente en relación con la calidad de servicio esperado y recibido del Servicio Autónomo Municipal de Administración Tributaria (SAMAT)

Para detectar el nivel de expectativas y la percepción de los contribuyentes marabinos relacionada con el servicio que esperan recibir en el SAMAT; se les consultó, antes de acceder a realizar sus requerimientos, acerca de la importancia que le otorgan a cada una de las dimensiones del servicio planteadas en el instrumento de la investigación. Mientras que la percepción se determinó aplicando el instrumento al momento en que los contribuyentes marabinos abandonaban la institución, luego de recibido el servicio, en este momento, se les invitó a responder si estaban de acuerdo con cada una de las aseveraciones referentes a cada una de las dimensiones del servicio planteadas.

\section{- Elementos tangibles}

Los resultados, fueron muy claros, y pueden visualizarse en la Tabla 1 las expectativas y en la Tabla 2 la percepción de los elementos tangibles.

En la Tabla 1, se evidencia como los contribuyentes marabinos $(92,97 \%)$ tienen altas expectativas de gestionar sus obligaciones tributarias en unas instalaciones cómodas, donde haya presencia de aires acondicionados, sillas suficientes y cómodas, televisión, ambiente decorado adecuadamente, limpieza, acceso a baños limpios, entre otros.

Por otra parte, los contribuyentes consideran muy importante un fácil acceso por medio de transporte público $(82,23 \%)$ en tanto que una gran mayoría $(92,97 \%)$ manifestaron altas expectati- vas acerca del acceso vehicular y al uso de un estacionamiento adecuado.

El contribuyente marabino mostró altas expectativas con respecto a los elementos tangibles, tanto desde el punto de vista del estado de las instalaciones internas y externas del SAMAT $(92,60 \%)$; así como también, con respecto al uso de uniformes del personal $(92,60 \%)$; existencia de señalización tanto dentro como fuera del recinto de servicio para ubicar las distintas áreas que facilite las gestiones $(92,60 \%)$; entre otros aspectos.

Los resultados expuestos permiten señalar que las organizaciones tienen el deber de desarrollar una oferta integral que genere una serie de atributos capaces de brindarle valor al cliente, lo cual le otorgara una ventaja competitiva sostenible en el tiempo.

Zeithaml y Bitner (2002), enfatizan que la apariencia de las instalaciones físicas transmite representaciones físicas o imágenes del servicio que los clientes, y en particular los nuevos, utilizarán para evaluar la calidad. Por lo tanto es fundamental e imprescindible que el SAMAT, tome muy en cuenta el estado de sus instalaciones físicas internas.

En este sentido, en la Tabla 2, se observa la evaluación que hicieron los contribuyentes encuestados, una vez recibido el servicio. En general, los usuarios del SAMAT tuvieron una percepción positiva acerca de: gestionar sus obligaciones tributarias en unas instalaciones cómodas (85,93\%); fácil acceso por medio de transporte público $(51,10 \%)$; buen estado del edificio, así como de la jardinería y la limpieza externa de las instalaciones del SAMAT $(94,46 \%)$; uso de uniforme por parte de los empleados $(94,09 \%)$; la 


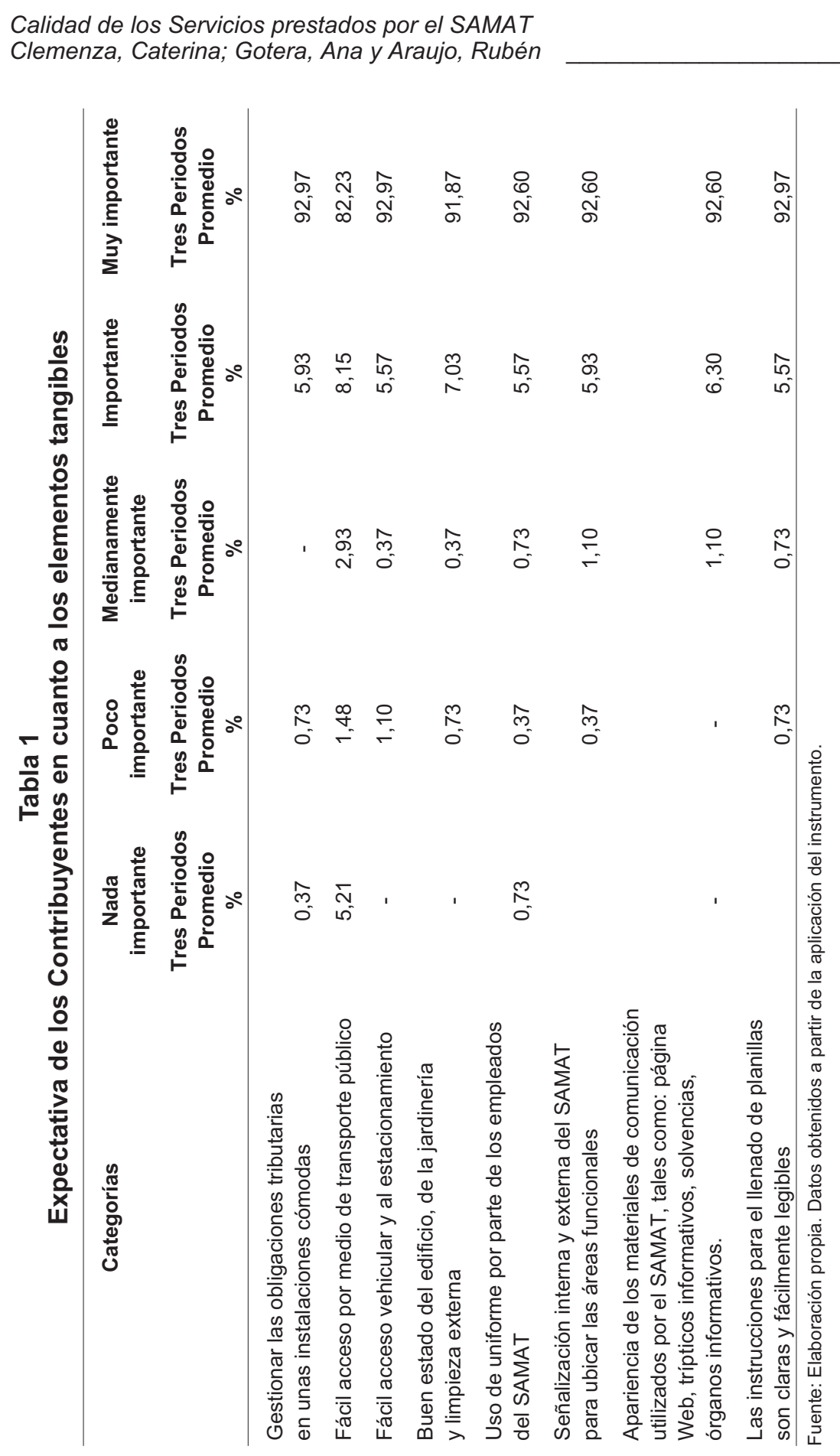


Revista Venezolana de Gerencia, Año 15, No. 49, 2010

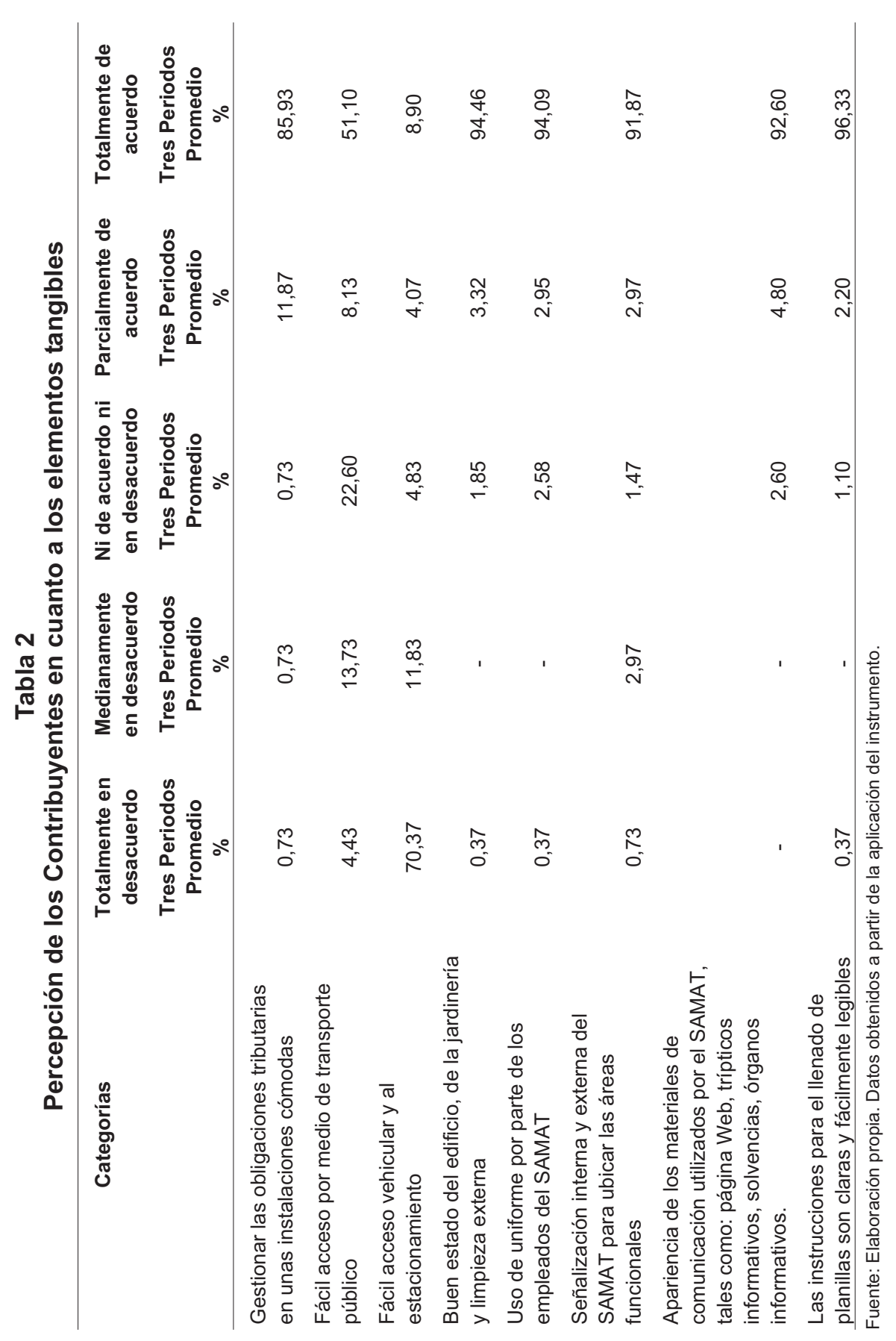


Calidad de los Servicios prestados por el SAMAT

Clemenza, Caterina; Gotera, Ana y Araujo, Rubén

existencia de señalización tanto en el área interna como en la externa del SAMAT $(91,87 \%)$; apariencia de los materiales de comunicación utilizados $(92,60 \%)$ y acerca de instrucciones claras y fácilmente legibles para el llenado de planillas $(96,33 \%)$. En cuanto al fácil acceso vehicular y estacionamiento $(70,37 \%)$ la muestra manifestó encontrarse totalmente en desacuerdo detectándose claramente una percepción negativa.

\section{- Dimensiones Intangibles}

Empatía: De acuerdo a los resultados obtenidos, en la Tabla 3 se observa que los contribuyentes del SAMAT, poseen altas expectativas en todos los indicadores planteados. Así, la atención personalizada $(91,11 \%)$; ser atendido amablemente $(91,48 \%)$, que conozcan sus necesidades $(92,97 \%$ ) y el manejo de quejas con honestidad y equidad $(93,79 \%)$ fue evaluado como muy importantes.

En cuanto a la percepción del servicio, la Tabla 4 muestra los resultados obtenidos, destacándose la percepción positiva manifestada en la atención personalizada $(74,44 \%)$; aún cuando se debe prestar atención al grupo de contribuyentes que manifestaron una opinión contraria $(25,56 \%)$ al momento de tomar decisiones en la mejora del servicio.

Resultados similares se evidencias en las otras categorías de análisis como: ser atendido amablemente $(74,78 \%)$, que conozcan sus necesidades $(77,40 \%)$ y el manejo de quejas con honestidad y equidad $(86,30 \%)$.

La percepción no solo depende de estímulos físicos, sino también de los relacionados con el entorno y las circunstancias del individuo. La palabra clave en la definición de percepción, es el indivi- duo. Mientras mayor sea el grado de participación física de los clientes en el proceso de servicio, mas probabilidades hay de que el personal del servicio, el equipo y las instalaciones constituyan un papel de vital importancia en la experiencia de servicio.

Estos rasgos influyen en le servicio prestado por el SAMAT, si se considera que esta es una institución que debe buscar consolidar una cultura tributaria en los contribuyentes marabinos, para lo cual la actitud del personal es fundamental, ya que ellos son corresponsables de que el contribuyente se sienta bien atendido, brindarle apoyo e informarle sobre el pago de sus tributos y el destino de los mismos, así como también ayudarlos a conocer sus obligaciones tributarias municipales.

Seguridad: Al preguntarle a los contribuyentes acerca de cómo clasificaban sus expectativas en cuanto a la existencia de una buena comunicación interdepartamental (recepción, catastro, entre otros) que le brinden solución a sus problemas, el 93,33\% de la muestra indicó tener altas expectativas. De igual manera esperan que se les brinde información asertiva que responda sus inquietudes en un lenguaje que ellos puedan entender fácilmente $(94,06 \%)$; así como el suministro de información en caso de retraso $(94,08 \%)$ (Ver Tabla 5).

Representando la comunicación un factor clave, para inspirar seguridad a los usuarios, para estos resulta un factor muy importante el hecho de la existencia de una buena comunicación tanto directa de empleado / usuario como interna entre los mismos departamentos, todo esto para lograr gestionar sus obligaciones de una manera rápida y segura. 
Revista Venezolana de Gerencia, Año 15, No. 49, 2010

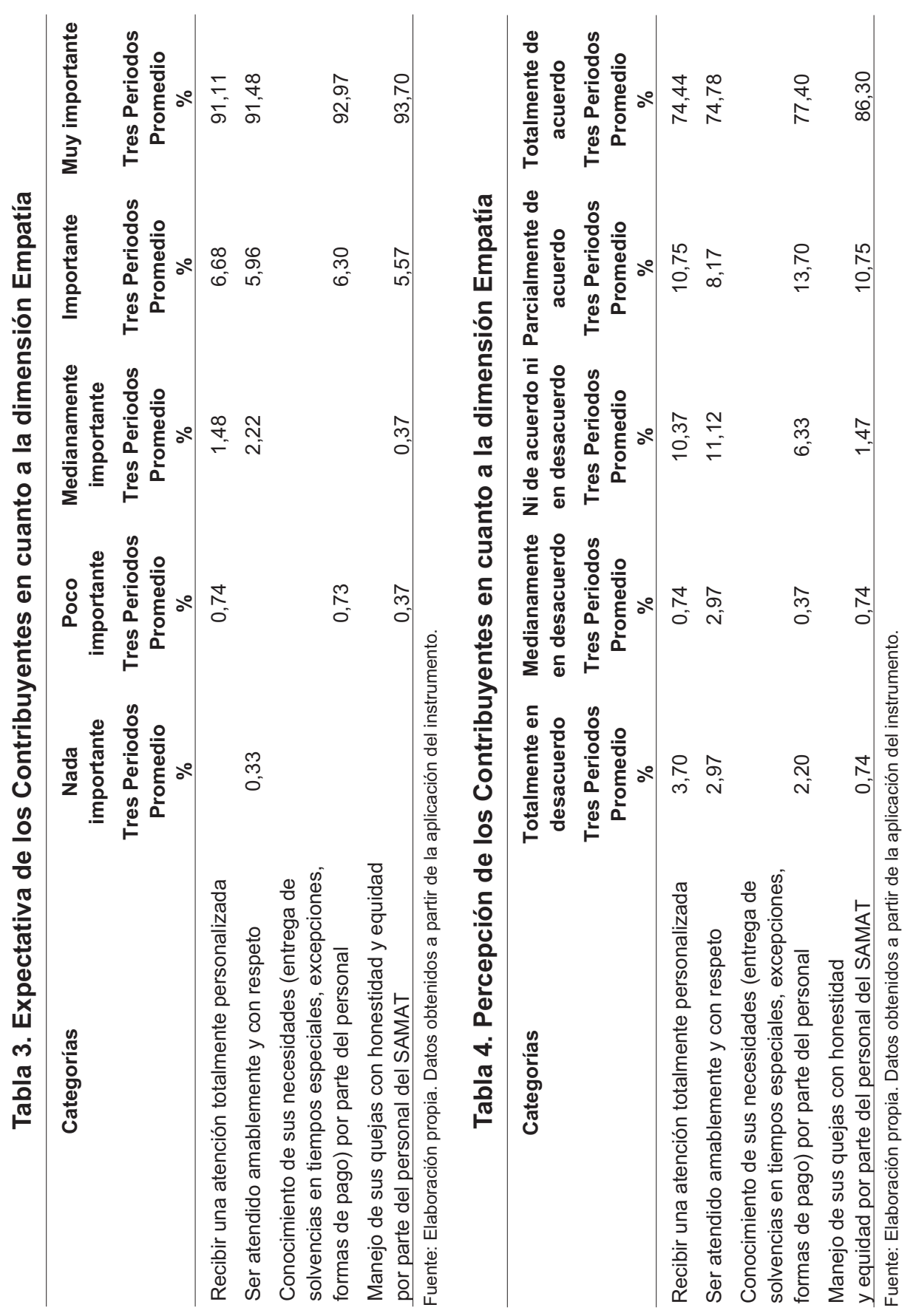


Calidad de los Servicios prestados por el SAMAT

Clemenza, Caterina; Gotera, Ana y Araujo, Rubén

Tabla 5

Expectativa de los contribuyentes en cuanto a la dimensión seguridad

\begin{tabular}{|c|c|c|c|c|c|}
\hline \multirow[t]{2}{*}{ Categorías } & $\begin{array}{c}\text { Nada } \\
\text { importante }\end{array}$ & $\begin{array}{c}\text { Poco } \\
\text { importante }\end{array}$ & $\begin{array}{l}\text { Medianamente } \\
\text { importante }\end{array}$ & Importante & $\begin{array}{c}\text { Muy } \\
\text { importante }\end{array}$ \\
\hline & $\begin{array}{c}\text { Tres } \\
\text { Periodos } \\
\text { Promedio } \\
\% \\
\end{array}$ & $\begin{array}{c}\text { Tres } \\
\text { Periodos } \\
\text { Promedio } \\
\% \\
\end{array}$ & $\begin{array}{c}\text { Tres } \\
\text { Periodos } \\
\text { Promedio } \\
\% \\
\end{array}$ & $\begin{array}{c}\text { Tres } \\
\text { Periodos } \\
\text { Promedio } \\
\% \\
\end{array}$ & $\begin{array}{c}\text { Tres } \\
\text { Periodos } \\
\text { Promedio } \\
\%\end{array}$ \\
\hline $\begin{array}{l}\text { Existencia de una buena } \\
\text { comunicación } \\
\text { interdepartamental que } \\
\text { le ayude a solucionar sus } \\
\text { problemas }\end{array}$ & - & 0,37 & 0,73 & 5,57 & 93,33 \\
\hline $\begin{array}{l}\text { Información asertiva que le } \\
\text { responda sus inquietudes } \\
\text { en un lenguaje que usted } \\
\text { pueda entender fácilmente }\end{array}$ & - & 0,74 & 0,37 & 4,81 & 94,08 \\
\hline $\begin{array}{l}\text { Se le informa en el caso de } \\
\text { algún retraso en su } \\
\text { solicitud }\end{array}$ & 0,74 & 0,37 & 4,81 & - & 94,08 \\
\hline
\end{tabular}

Fuente: Elaboración propia. Datos obtenidos a partir de la aplicación del instrumento.

En cuanto a la percepción de la dimensión seguridad en el servicio, los resultados obtenidos, destacan la percepción positiva manifestada en la existencia de una buena comunicación interdepartamental que le ayuda a solucionar sus problemas $(65,18 \%)$, se les brinda Información asertiva que responda sus inquietudes en un lenguaje de fácil comprensión $(78,53 \%)$ y recibe información en el caso de algún retraso en su solicitud (45,20\%).

Responsabilidad: Esta dimensión lleva a la organización a asegurarse de observar el proceso de la prestación del servicio y del manejo de las solicitudes desde el punto de vista del cliente y no desde su propio punto de vista. Cuando las organizaciones desean diferenciarse por su responsabilidad, necesitan contar con el personal de contacto correcto y muy bien entrenado y capacitado en el departamento de servicio al cliente (Zeithmal y Bitner, 2002).

En este orden de ideas, se observa las altas expectativas manifestada por los contribuyentes en cuanto a la prontitud en la prestación del servicio (93,94\%); en la voluntad de colaboración por parte del personal $(93,70 \%)$ y en obtener un servicio flexible y personalizado cuando se amerite $(93,71 \%)$.

En cuanto a la percepción de los contribuyentes con respecto al servicio ofrecido, se tiene que esta se mantuvo en un rango superior al $50 \%$, denotando una percepción positiva en cuanto a la dimensión analizada (ver Tabla 6).

Confiabilidad o Fiabilidad: De acuerdo a los resultados se evidencia que los contribuyentes del SAMAT, po- 
Tabla 6

Percepción de los contribuyentes en cuanto a la dimensión responsabilidad

\begin{tabular}{|c|c|c|c|c|c|}
\hline Categorías & $\begin{array}{c}\text { Totalmente en } \\
\text { desacuerdo } \\
\text { Tres } \\
\text { Periodos } \\
\text { Promedio } \\
\% \\
\end{array}$ & $\begin{array}{c}\text { Medianamente } \\
\text { en desacuerdo } \\
\text { Tres } \\
\text { Periodos } \\
\text { Promedio } \\
\% \\
\end{array}$ & $\begin{array}{c}\text { Ni de acuerdo ni } \\
\text { en desacuerdo } \\
\text { Tres } \\
\text { Periodos } \\
\text { Promedio } \\
\%\end{array}$ & $\begin{array}{c}\text { Parcialmente } \\
\text { de acuerdo } \\
\text { Tres } \\
\text { Periodos } \\
\text { Promedio } \\
\%\end{array}$ & $\begin{array}{c}\text { Totalmente } \\
\text { de acuerdo } \\
\text { Tres } \\
\text { Periodos } \\
\text { Promedio } \\
\% \\
\end{array}$ \\
\hline $\begin{array}{l}\text { Prontitud en } \\
\text { la prestación } \\
\text { del servicio }\end{array}$ & 1,84 & 4,45 & 14,80 & 23,73 & 55,18 \\
\hline $\begin{array}{l}\text { Voluntad de } \\
\text { colaboración } \\
\text { por parte del } \\
\text { personal }\end{array}$ & 1,10 & 0,37 & 11,12 & 19,27 & 68,15 \\
\hline $\begin{array}{l}\text { Servicio } \\
\text { flexible y } \\
\text { personalizado } \\
\text { cuando se } \\
\text { amerite }\end{array}$ & 2,60 & 2,57 & 12,97 & 24,83 & 57,03 \\
\hline
\end{tabular}

Fuente: Elaboración propia. Datos obtenidos a partir de la aplicación del instrumento.

seen altas expectativas en cuanto a que sus tributos sean calculados por personas expertas en la materia (94,08\%). De igual manera, consideran muy importante el pago y cálculo de sus impuestos vía electrónica $(60,74 \%)$. En el resto de los indicadores se mantiene el mismo comportamiento. Así, el suministro de información asertiva y oportuna (93,71\%); y la información acerca de los destinos de sus tributos $(94,08 \%)$ fueron evaluados como muy importante.

Los usuarios del SAMAT tuvieron una percepción positiva acerca de la confianza en el cálculo de sus obligaciones tributarias $(90,38 \%)$, y al suministro de información asertiva y oportuna $(85,58 \%)$; mientras que evidenciaron poca confianza en el cálculo y pago vía electrónica
$(39,63 \%)$ y la información acerca de los destinos de sus tributos $(31,83 \%)$ (ver Tabla 7).

c. Diferencia entre las expectativas y la percepción del servicio prestado por el Servicio Autónomo Municipal de Administración Tributaria (SAMAT).

Para Zeithaml y Bitner (2002), los clientes pueden reflejar sus opiniones sobre la calidad de los servicios, contrastando lo que ellos esperaban (expectativas) antes de recibir el servicio con su experiencia real del servicio (percepción). La calificación del servicio será igual a las percepciones que tienen de lo que recibieron menos las expectativas que tienen respecto a lo que pensaban que debieron haber recibido. 
Calidad de los Servicios prestados por el SAMAT

Clemenza, Caterina; Gotera, Ana y Araujo, Rubén

Tabla 7

Percepción de los contribuyentes en cuanto a la dimensión confiabilidad

\begin{tabular}{|c|c|c|c|c|c|}
\hline Categorías & $\begin{array}{c}\text { Totalmente en } \\
\text { desacuerdo } \\
\text { Tres } \\
\text { Periodos } \\
\text { Promedio } \\
\% \\
\end{array}$ & $\begin{array}{c}\text { Medianamente } \\
\text { en desacuerdo } \\
\text { Tres } \\
\text { Periodos } \\
\text { Promedio } \\
\% \\
\end{array}$ & $\begin{array}{c}\text { Ni de acuerdo ni } \\
\text { en desacuerdo } \\
\text { Tres } \\
\text { Periodos } \\
\text { Promedio } \\
\%\end{array}$ & $\begin{array}{c}\text { Parcialmente } \\
\text { de acuerdo } \\
\text { Tres } \\
\text { Periodos } \\
\text { Promedio } \\
\% \\
\end{array}$ & $\begin{array}{c}\text { Totalmente } \\
\text { de acuerdo } \\
\text { Tres } \\
\text { Periodos } \\
\text { Promedio } \\
\% \\
\end{array}$ \\
\hline $\begin{array}{l}\text { Confianza en e } \\
\text { cálculo de sus } \\
\text { obligaciones o } \\
\text { tributos }\end{array}$ & 0,37 & 1,48 & 3,71 & 4,06 & 90,38 \\
\hline $\begin{array}{l}\text { Confianza en e } \\
\text { cálculo y pago } \\
\text { de sus } \\
\text { impuestos vía } \\
\text { electrónica }\end{array}$ & 1,10 & $4,10 \%$ & 37,40 & 17,77 & 39,63 \\
\hline $\begin{array}{l}\text { Suministro de } \\
\text { información } \\
\text { asertiva y } \\
\text { oportuna }\end{array}$ & 0,37 & 1,48 & 7,01 & 5,57 & 85,58 \\
\hline $\begin{array}{l}\text { Información } \\
\text { acerca del } \\
\text { destino de sus } \\
\text { tributos }\end{array}$ & 7,03 & $18,53 \%$ & 16,30 & 26,30 & 31,83 \\
\hline
\end{tabular}

Fuente: Elaboración propia. Datos obtenidos a partir de la aplicación del instrumento.

Cuando se satisfacen las expectativas de los clientes o usuarios, las percepciones y las expectativas son iguales, y en consecuencia, califican el servicio como satisfactorio. Cuando la empresa no satisface las expectativas de los clientes (si por ejemplo, sus expectativas son mas altas que el servicio que realmente les fue ofrecido), la calificación será negativa y el cliente clasificara el servicio como no satisfactorio. Si en caso contrario el servicio sobrepasa las expectativas de los clientes o usuarios, la clasificación será positiva y el cliente calificara el servicio como superior.
Cuanto mas grande sea la brecha, mas alejadas estarán las percepciones de las expectativas y mas baja será la evaluación de la calidad del servicio. Al contrario, cuanto menor sea la brecha mayor será la evaluación de la calidad del servicio (Duque, 2005; Hoffman y Bateson, 2002; Zeithaml y Bitner, 2002).

En los Gráficos 2 y 3, se evidencia la calificación realizada por los contribuyentes marabinos como no satisfactoria ya que el resultado de la percepción $(4,38)$ menos las expectativas $(4,89)$ resultó - 0,53. Específicamente se tienen: 


\section{Gráfico 2}

Percepción versus expectativas en cuanto a los elementos tangibles e intangibles

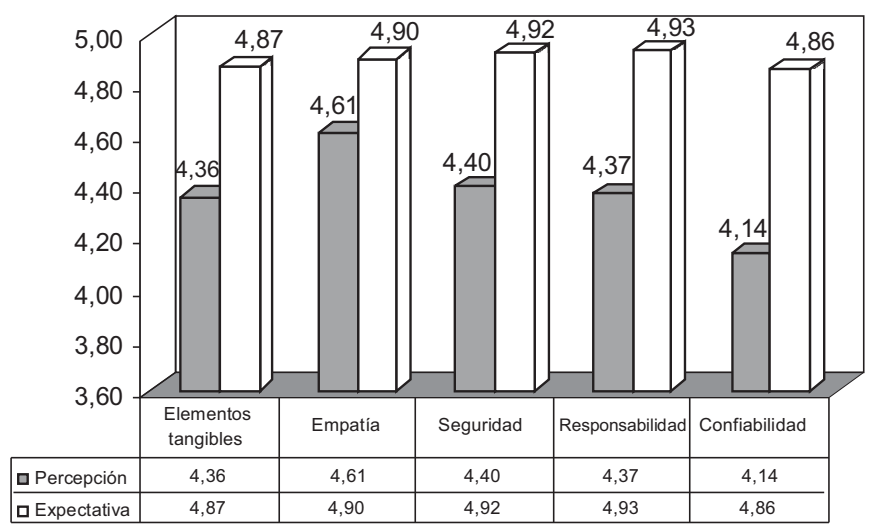

Fuente: Elaboración propia a partir del procesamiento del instrumento aplicado.

\section{Gráfico 3}

Calidad de servicio en cuanto a los elementos tangibles e intangibles del servicio

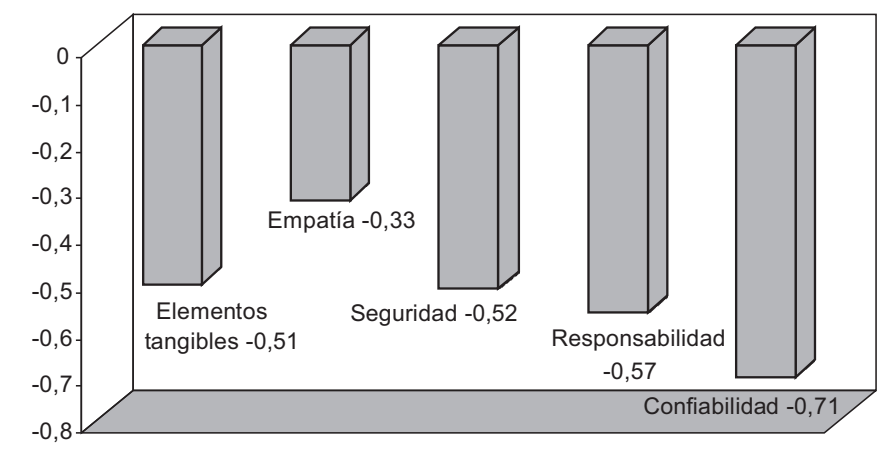

Fuente: Elaboración propia.

\section{Conclusiones}

En torno a estos resultados, se observa como el cliente se comporta en el ambiente del servicio al momento de acudir a una organización en particular con el propósito de adquirir bienes o servicios. En este sentido, los usuarios del SAMAT pueden reaccionar acercándose o evadiendo el ambiente donde se presta el servicio, por lo que entenderlos y orientar a la institución hacia ellos, se convierte en 
Calidad de los Servicios prestados por el SAMAT

Clemenza, Caterina; Gotera, Ana y Araujo, Rubén

una estrategia necesaria para generar un acercamiento del contribuyente y solventar los inconvenientes que atentan contra el servicio, en la recaudación tributaria municipal.

Al analizar la diferencia entre las percepciones y las expectativas (P-E) es preciso señalar que las categorías estudiadas presentan desviaciones negativas y por tanto son los aspectos que SAMAT deberían tratar de mejorar en función de satisfacer a sus usuarios. Por tanto, los usuarios del servicio, no se encuentran satisfechos con la calidad del servicio ofrecida, ya que resultó menor la puntuación del servicio percibido con respecto al esperado.

Para el SAMAT, no solo los elementos tangibles de la institución deben desempeñar un papel muy importante en el proceso de relación entre el personal y los contribuyentes, si no debe considerar en el mismo orden jerárquico los aspectos intangibles (empatía, seguridad, confianza, responsabilidad). Ya que a través de este proceso de socialización se proyecta una imagen positiva y congruente entre lo que el usuario espera y percibe y lo que la institución es capaz de ofrecer.

Por lo general, el servicio que recibe el contribuyente es el resultado de una cadena de servicios interrelacionados, donde no sólo las evidencias físicas del mismo son evaluadas por éstos, sino que además contemplan el carácter intangible de los servicios.

\section{Referencias Bibliográficas}

Berry, Leonard (2004). Un buen servicio ya no basta. Ediciones DEUSTO. España.

Cobra, Marcos (2001). Marketing de Servicios. Segunda Edición. Editorial Mc Graw Hill. Colombia.

Cotlle, David (1991). El servicio centrado en el cliente. Ediciones Díaz de Santos S.A, Madrid, España.

Duque Oliva (2005). Revisión del concepto de calidad del servicio y sus modelos de medición. En Revista Innovar. Enero-Junio No. 24 Págs. 64-77. Colombia.

Franco, Gilda (2003). Validación de SERVQUAL en una institución pública mexicana. En revista UPIICSA. Tecnología, Ciencia y Cultura. Año XI. Vol. 5. No.33. Septiembre-diciembre. Págs. 25-28- México.

Grande, Idelfonso (2000). Marketing de los Servicios. Tercera Edición. ESIC Editorial. Madrid, España.

Hoffman, Douglas y Bateson, John (2002). Fundamentos de Marketing de Servicios. Conceptos, estrategias y casos. Segunda Edición. Editorial Thomson. México.

Kotler, Philip; Armstrong, Gary; Cámara D. y Cruz I. (2004) (2004). Marketing. Décima Edición. Editorial Prentice- Hall. México.

Lovelock Christopher (1997). Mercadotecnia de Servicios. Tercera Edición. Editorial Prentice-Hall. México.

Zeithaml, Valarie y Bitner, Mary (2001). Marketing de Servicios. Segunda Edición. Editorial Mc Graw Hill. México. 